\title{
The Analysis for the Survey of Construction Status of the Class Tutor in the University
}

\author{
Cao Jianshu Hou Jun \\ (School of International Education, Wuhan University of Technology, Wuhan, Hubei, 430070)
}

\begin{abstract}
:
Following the development of popularization of higher education, and increase of the student recruitment of the university, as well as the implementation of the policy of credit system, the previous mode relies only on assistant for political and ideological work which is extensive management, as well as superficial and simplified work. To avoid the short-coming of the management for the mode of assistant for political and ideological work and class tutor, there are many universities which are exploring the new mode of personnel training which could combine their own situation, for example to set up class tutor in the undergraduate class. Class tutors are regarded as the direct guides, educators and managers for the study and living of the university student and it is worth to have more researches and exploration for their effects of the work, performances for their education and management of the students, long-term effect mechanism to be formed or not for class tutor in the university, etc.
\end{abstract}

Key words: Class tutor; long-term effect mechanism; measure

Independent innovative research fund: Supported by" the Fundamental Research Funds for the Central Universities"

\section{The objective and methods of survey}

Following the development of popularization of higher education, and increase of the student recruitment of the university, as well as the implementation of the policy of credit system, the previous mode relies only on assistant for political and ideological work which is extensive management, as well as superficial and simplified work. In order to avoid the short-coming of the management for the mode of assistant for political and ideological work and class tutor, there are many universities which are exploring the new mode of personnel training which could combine their own situation, for example to set up class tutor in the undergraduate class. So we start to have the survey for current effect of class tutor in some universities of Wuhan city.

The survey is combined by two parts which are questionnaire and structured interview. The questionnaire is to have investigation with students and their class tutors at the same time, which is mainly to know the effect of class tutor system and attitude of recognition from the student as well as class tutors to the system from the quantitative perspective. In case of sampling error, the total population of this survey was estimated to be 1800 persons, and there were 1650 pieces of questionnaires to be sent, later there were 1572 pieces of returned questionnaires to be effective, the total effective rate reached $95.3 \%$. In addition, the research group had structured interviews with 186 class tutors from universities of Wuhan city, and to know more about the difficulties and problems in the process of implementation of the class tutor system, and to listen to their opinions and suggestions for the long-term construction mechanism of class tutor in the university, so as to provide theoretical and practical support for the innovative personnel training mode of university.

\section{The current situation of implementation of class} tutor system

The questionnaire mainly focus on some perspectives like how much the students know about their class tutors, and the students' attitude to the system, detailed effect of implementation of class tutor system, and etc. so as to know the construction status of the long-term mechanism of class tutor. In order to better explore the long-term mechanism of class tutor, the questionnaire also designed related topics about the expectation and suggestion from the students to their class tutors. 


\subsection{How much the students know about their class tutor}

\begin{tabular}{ccc} 
Understanding level & Frequency & Total percentage $(\%)$ \\
\hline Very much & 45 & 2.9 \\
Big part of & 280 & 17.8 \\
A little & 520 & 33.1 \\
Barely & 493 & 31.4 \\
Never & 228 & 14.5 \\
\hline In total & 1572 & 100.0
\end{tabular}

Table 1: The percentage of students know about their class tutor

From table 1, the public would make the following conclusion based on the answers that students know less about the class tutor system. $79 \%$ of the students being interviewed showed that they don't much about the system (including knows a little, barely knows, and never knows), which means the effect of implementation of class tutor system is not very good. There has been a while since people start to explore the class tutor system in the academic world, but its implementation has not been fully recognized, which means the popularization and deeper exploration of class tutor system still have a long way to go.

\subsection{The Satisfaction of the students to the class tutor}

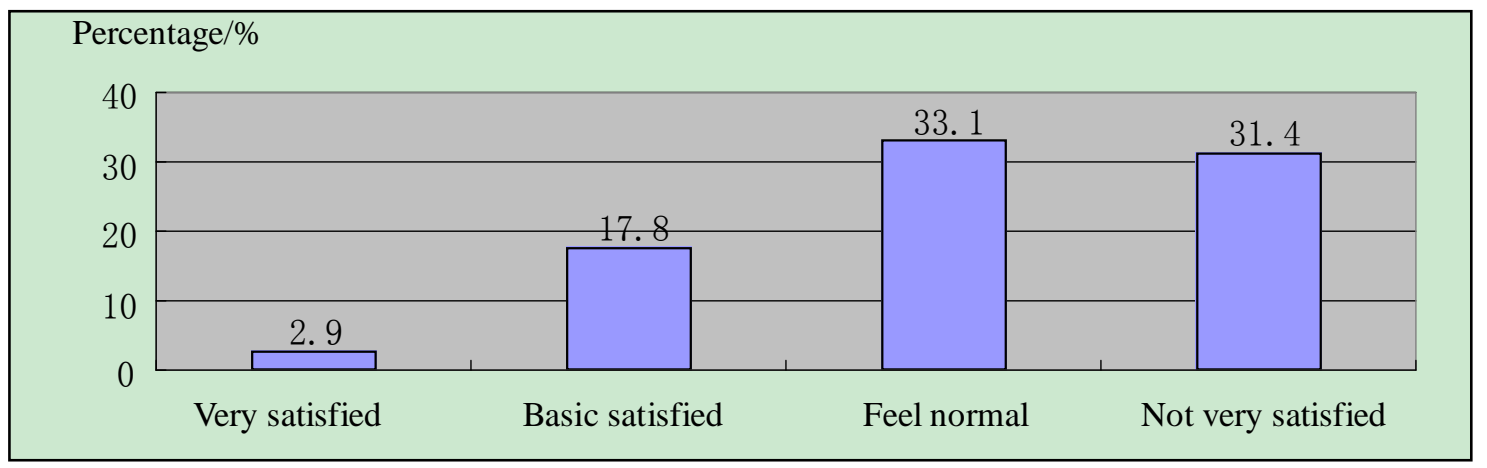

Fig. 1: The satisfaction of the students to the class tutor

From figure 1, most of the students were satisfied with their class tutors. And only $8 \%$ of the students being interviewed are not satisfied, and around $65.7 \%$ of the students being interviewed are basically satisfied. In the survey of "Do you think class tutor is necessary", there were $96.3 \%$ of students who keep positive answer. This result is closely connected with the recognition of students to current class tutor system. Though most of the students are not very familiar with class tutor system, but they still appear strong sense of identity and coherence for the necessity of existence of class tutor system, as well as the acceptance of the work of class tutor system.

\subsection{The problems existing in the implementation of class tutor system}

In order to find the problem existing in the process of implementation of class tutor system, we choose to have research in the fields of communication frequency between students and class tutors, and the relation between them.

Percentage/\%

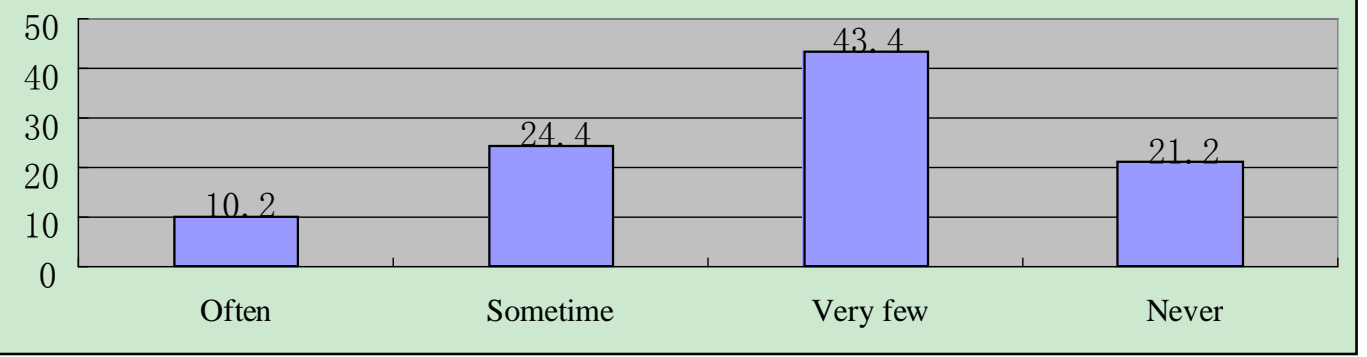

Fig. 2: Communication frequency between students and class tutors 


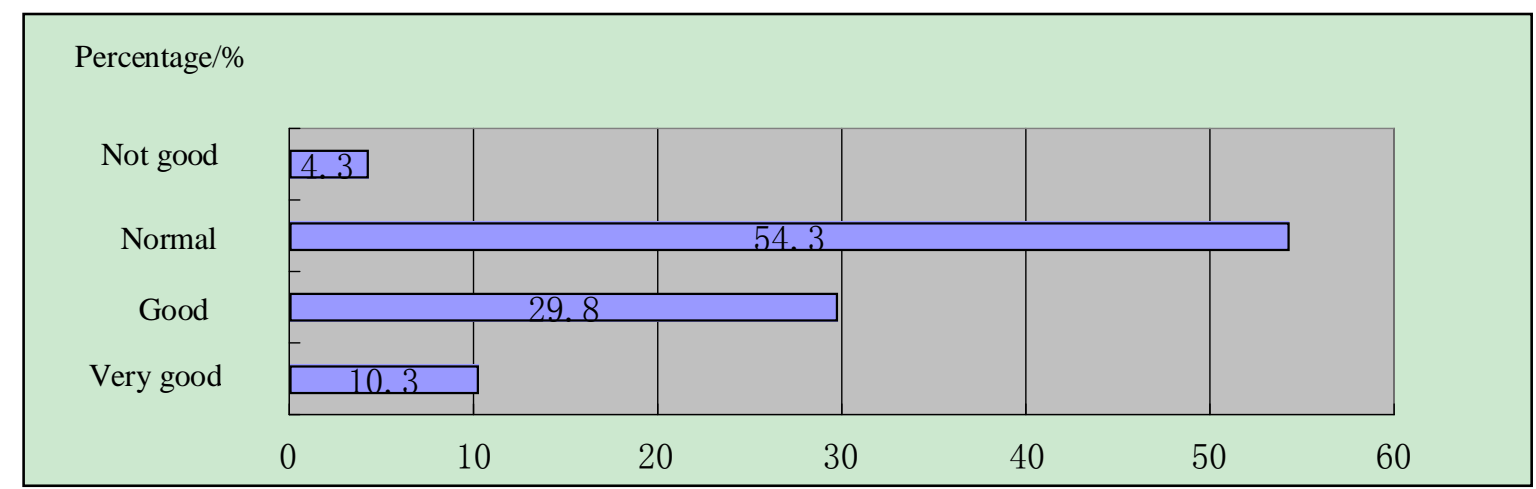

Fig. 3: Relations between students and class tutors

By the research in the fields of communication frequency between students and class tutors, and the relation between them, we could know the detailed status of implementation of class tutor system. From Figure 2, 3, we could see that the communicate frequency is rather low, and the evaluation for the relation between students and class tutors is not very good. There were $64.6 \%$ of students who communicate less with their class tutors (including occasionally and never), and only $40.1 \%$ of students thought they have good relationship with class tutors, and most of them thought their relationship is just ok. All these above could see that the class tutor system needs to be perfect, and the class tutor system has the problem in inefficient communication and not effective connection, etc. during the implementation.

\subsection{Suggestions from the students to the construction of class tutor system}

In the questionnaire, we designed some questions to help students to give suggestions to the construction of class tutor system so as to get first-hand materials for our analysis to solve the problem.

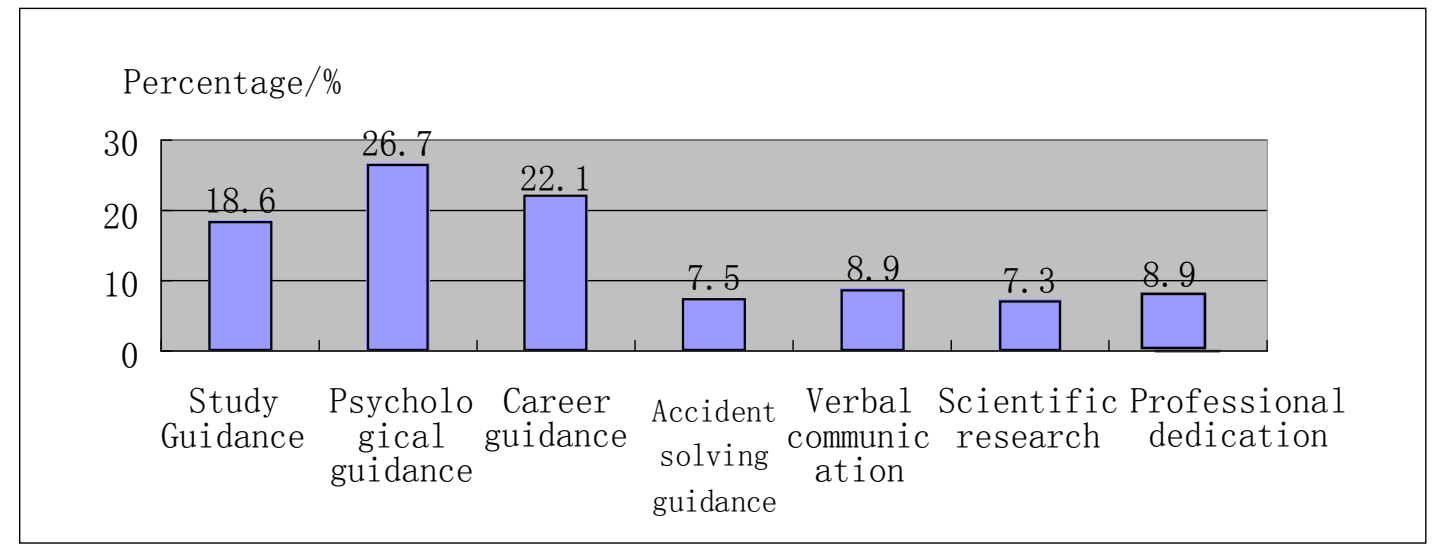

Fig. 4: What kind of training needs to be provided to class tutor?

Percentage/\%

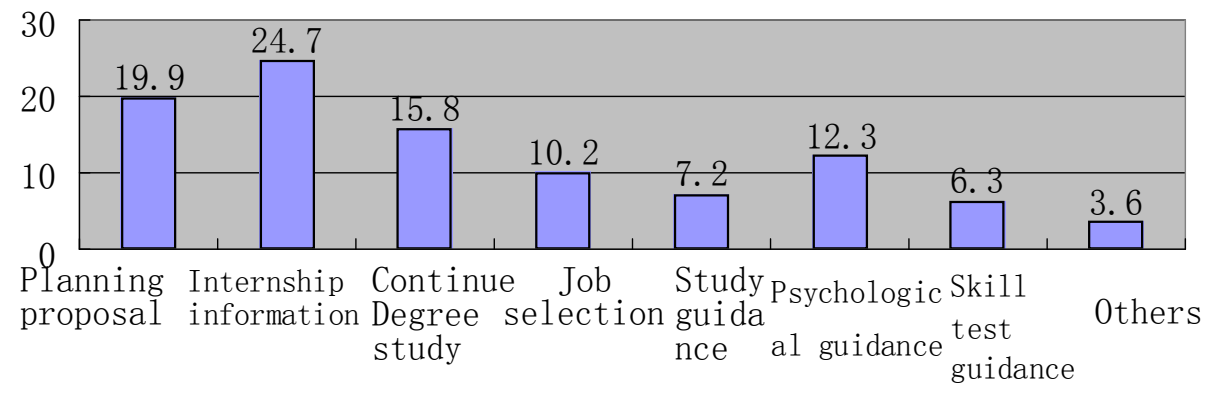

Fig. 5: What kind of help you want from the class tutor?

In order to build long-term mechanism of class tutor system, to serve the students better, we designed related questions on what kind help from the class tutors, and the expectation from the class tutors for the training. 
From the Figure 4 and 5, we could see that no matter training or help, the requests for the class tutors by the students had appeared the trend of diversified, complicated and decentralized, their needs are multi-leveled, which means higher needs for the class tutors of the university, and see the long way and importance of long-term mechanism for team construction of class tutors.

\section{The existing problem during the construction of} class tutor system and its analysis

From collected datum of questionnaire and information through the interview with class tutors, it is not very optimism for the status of long-term construction of class tutor system in the university, and the problems mainly appear in three ways. One problem is neither the students nor their class tutors are very familiar with the concrete responsibilities, functions, and regulations; one is that the special value of class tutor which is focusing on "students guiding" could not be revealed, and there is not obvious differences compared with head teacher and counselor, and the last one is that class tutors are not very active nor very satisfied with their work, which leads to insufficient input into their work. The reason caused for the problems above are multiple.

\subsection{The management mode has not formed for the combination of class tutor, head teacher and counselor}

The responsibilities are not clear among class tutor, head teacher and counselor, which could not make into the whole, or into the management mode of combination of class tutor, head teacher and counselor. From the teachers to the students, most of them are not clear with the difference and connection among them, that is main cause that why the class tutor system could not motivate the initiative of the teachers, nor get the full recognition by the teachers and students. Theoretically, the objective of duty of class tutor, head teacher and counselor is to teach, manage, serve and cultivate, there are coordination and different focus among them. ${ }^{\text {[1] }}$ In the investigation, we found that the work of class tutor, head teacher and counselor are generally separate, disconnected, and not strong in coordination, which could not effectively use their advantages to help, guide or manage students according to students' needs. Since the students are not clear of the difference of class tutor, head teacher and counselor, so they don't know who to be looking for when meeting the problem. The counselors normally have their office, and regulated working time, and meet student regularly, so students would always go to ask counselor when they meet problem, which increase the work load of counselor, and could not effective use the function of head teacher and class tutor. In the other way, the questions of the students could not be guided in the professional and specific way. That is why it is required to build the management mode of combination of class tutor, head teacher and counselor so as to provide better service to the students.

\subsection{There is no complete mechanism of assessment, stimulation, and training for the class tutors of undergraduates}

The university has not set up regulations for the new-born class tutor, nor formed complete mechanism of assessment and stimulation. Since the class tutor system is a new thing, and not all the class tutors could fully understand the seriousness and long benefits of work of class tutor system ${ }^{\lfloor 2\rfloor}$. So it is extreme important to have management regulations for assessment and stimulation that has not yet taken it's' responsibilities, which appears mainly in the following aspects. One is that the measures of stimulation are too simple, and it is not to stimulate according to the needs. The teachers of the university are the group which has strong needs of self-fulfillment. Though the class tutors of undergraduate are different from the teachers to be familiar with teaching and scientific research, the class tutors also focus on the self-fulfillment like teachers, and the current measurement is very difficult to enhance the working passion of the teachers. One is that the assessment regulation is not very scientific nor objective, most of the assessment would focus on qualitative short target, not on quantitative long term target. And the assessment procedure is lack of performance feedback, which leads to only stay on the outside not inside. The last one is lacking of effective training mechanism of class tutor. According to the survey, many class tutors never have the pre-training before the work. Since the position of class tutor is part-time carried by professional teachers, which don't have trainings of systematic mental health education for students, skills in dealing with interpersonal relationship, 
nor class construction, therefore would meet some difficulties in the work.

\subsection{There is no enough academic environment in the university, and the culture construction of campus it not enough}

In the traditional cultivation mode of undergraduate of the university in China, the students have teachers in the classroom, have counselors after class, and the class tutors would fill the blank between students' affairs and teaching affairs in some way. The class tutors are mainly focusing on "study guidance", which means to guide the students in the professional way, help students to cultivate their learning ability, especially self-study ability, most important is to guide the students to have innovative study, thinking and practice in the professional area. ${ }^{[3]}$ Following the recruitment enlargement of the higher education institutes, it is becoming more obvious that the trend for marketing the undergraduates education, and fickleness from the society has started to spread in the campus, the students are more and more focusing on techniques and knowledge which has more effects in the short-term, and it has become less and less for students to pursue science, neither to have enough academic environment in the university. For example, "Sound in Morality, Broad in Learning and Pursuing Excellence" and many excellent campus cultures have been only used more like slogan of the university to let the students know, but not being used to set up related measures to take inside the regular educational management of students, which would help the students to transfer the campus cultures into their own values, so as to form the real campus cultures.

4.The strategies to improve the formation of long-term mechanism of class tutors system in the higher education institutes

\subsection{To set up high efficient management mode of combination of class tutor, head teacher and counselor}

In the stages of growth of students, counsel, head teacher and class tutor would make different roles, they are combined together to affect each other, support each other, and benefit each other, and to mutually finish the duty to cultivate students. Head teachers mainly focus on lower grade group, which have weaker ability of study and living. The ability of study which is required to be trained in the primary school and secondary school has been postponed to the university due to the short-coming of exam-oriented education, and the head teacher is the carrier who is responsible for the management to help student finish the transition from senior secondary school to the university. While the class tutors would face the higher grade group, so normally they have higher degree, and have more experience in teaching and scientific research, which could provide concrete guidance according to the study needs and characteristics of students. The core target of the class tutor's work is "study guidance", which mainly focuses on three parts, which includes to provide recognition guidance for the major, to help to make self-planning, to guide students to make innovation of sciences and technology as well as academic explore, to help to form ability of innovative study, thinking and practice, to have further self promotion. The counselor is required to guide along with the ideological and political education, use interesting examples into the students party building and league building activities, general education management and service, as well as after class activities. Class tutor and head teacher are focusing on "point" during their work, and counselors are focusing on "facet" during their work, only "point" combined with "facet" could benefit each other, which leads the students' work into virtuous circles, and prevent to miss the right time or miss the right point to have the education.

\subsection{To complete regulation of training, evaluation,} and stimulation for class tutor system

First, the manager of university needs to have systematic pre-training for the class tutors. In the training, the importance of the work of class tutors need to be systematically illustrated so as to let the teachers to have full recognition of their job. The working handbook needs to be made, in which the main part of the work would be identified. The training also need to teach the teachers to how make mental health education for the students, how to handle interpersonal relationship, how to organize class construction, etc ${ }^{\mathbf{I} \mathbf{I}}$. Secondly, to introduce 360 degree performance feedback evaluation mechanism, in which is to evaluate the class tutor's work through different angles, like class tutor, higher authority, students of the class, and colleagues. And the evaluation content is 360 degree as 
well, like working measure, working attitude, communication skill, and leadership management capability. Finally, to set up combination incentive mechanism like material incentive, figure incentive, personalized requirements incentive. The teachers of the university normally have higher degree, so they are stronger to have the ambition to pursue self-esteem and self-realization compared to other groups in the society. So it is not only necessary to provide material rewards to the outstanding undergraduate class tutors according to the evaluation, but also provide awards like praise, title of honor and certificate through the spiritual way, so as to produce continuous and strengthened effect through figured incentive. Moreover, the managers are required to use different incentive ways to different class tutors based on their different needs so as to make the best effect.

\subsection{To set up advanced and excellent culture platform of university}

The deep implementation of the policy of credit system has made the student affairs more dynamic, microcosmic and complicated, the previous simple and united management based on class is not working very well, so it would be key factors to effectively implement undergraduate class tutor system, focusing on advanced and excellent university culture. The core working target of class tutor is "study guidance", which means to provide academic guidance and good academic environment are the premise for teachers and students both to have virtuous interaction. ${ }^{\mathbf{5} \mathbf{}}$ It is important to

actively build advance and excellent university culture, set up a good cultural platform of university, form noble value which could adapt to the society, and would be useful to the development of the students. It is human-based management, so as to let the teachers to devote themselves to work with happy mood, and to carry varies of regulations related to the students which could respect the individual difference, and encourage students to have full development according to their interests, capabilities and characteristics, and to become the future of the nation.

\section{Reference}

[1] Wang Meng, "Some thoughts on the work of class tutors,"Reform and Opening, pp.11, 2011.

[2] Wang Wei, Wang Yuhua, and Wang Shirong, "The Exploration and Practice for the Work of Undergraduate Class Tutor,"Journal of Higher Education Research (Chengdu), pp.3, 2011.

[3] Sun Lili, "The Importance of Class Tutors Following the Growth of University Students," The Road to Success, pp.25, 2011.

[4] Zhu Xuelian, Xiao Yue, and Zhou Ziming, "Approach to the Mechanism of Work Evaluation for Undergraduate Class Tutors of the University," Journal of Changchun University of Technology, pp.2, 2011.

[5] Luo Rong, and Zhang Kaibi, "Exploration of Working System Based on Study Guidance for Class Tutors in the University," Education Forum, 2011. also very important to involve the students inside the system, to carry on varies of regulations related to the teachers which could adapt to the psychological requirements of the teacher, to focus more on 